\title{
Review
}

Digestion

\section{Calprotectin in Daily Practice: Where Do We Stand in 2017?}

\author{
Fabrizia D'Angelo ${ }^{\mathrm{a}}$ Christian Felley $^{\mathrm{b}}$ Jean Louis Frossard ${ }^{\mathrm{a}}$ \\ ${ }^{a}$ Service of Gastroenterology and Hepatology, Geneva University Hospital, Geneva, and b Gastroenterology Clinic, \\ Clinique La Source Beaulieu, Lausanne, Switzerland
}

\section{Keywords}

Inflammatory bowel disease $\cdot$ Calprotectin $\cdot$ Biomarkers

\begin{abstract}
Background: To make a distinction between organic and functional disease is essential for gastroenterologists in their daily practice, but it may be challenging, given the variety and aspecificity of gastrointestinal symptoms among the general population. The clinician aim is to avoid diagnostic delay and to restrict unnecessary invasive and expensive exams. Summary: Faecal markers, in particular faecal calprotectin $(F C)$, have given proof of being reliable markers of intestinal inflammation with good clinical sensitivity. Calprotectin is useful in the differential diagnosis between inflammatory bowel disease and irritable bowel syndrome, as well as in the follow-up of inflammatory bowel disease patients and in predicting treatment response, with an excellent correlation with endoscopic activity. Its role in collagenous colitis and infectious colitis is less clear and still under investigation. Key Message: Despite the growing evidence supporting its use, many clinicians are uncomfortable in dosing FC, due to its low specificity and the variability of cut-off values. Indeed there are no clear guidelines about how to manage patients with intermediate levels of FC. The aim of this article is to review recent literature on calprotectin and its use. The strong points and the limits of FC measurement will be analysed, and a practical approach in the daily clinical routine will be proposed.

(c) 2017 S. Karger AG, Basel
\end{abstract}

\section{Introduction}

Gastrointestinal symptoms include a large variety of patients' complaints including diarrhoea, bloating, and abdominal pain and they represent a common reason to consult a gastroenterologist. The origin of these symptoms may include inflammatory bowel diseases (IBD) such as Crohn's disease (CD) and ulcerative colitis (UC), as well as collagenous colitis (CC), malignancy, infectious disease or functional disease (irritable bowel syndrome [IBS]). Differentiation between these conditions is necessary to avoid a diagnostic delay and to rapidly choose the correct treatment. In particular, it is essential to differentiate between functional and organic disease, which can be difficult to clarify solely on the base of the Rome III criteria that were specifically aimed to help the physician in charge of GI complaints. Actually, up to $40 \%$ of patients with IBD have the same presentation as patients with IBS [1]. This causes an important diagnostic delay, which can be up to 24 months for CD and 12 months for UC [2].

Even if in some cases patient history and physical examination may raise suspicion for a specific disease, physicians need complementary exams in the majority of clinical situations including laboratory tests, radiology or endoscopy with biopsy sampling.

Inflammatory serological markers such C-reactive protein (CRP), erythrocyte sedimentation rate (ESR) and leucocyte count can be considered a first screening tool.

\section{KARGER}

(c) 2017 S. Karger AG, Basel 


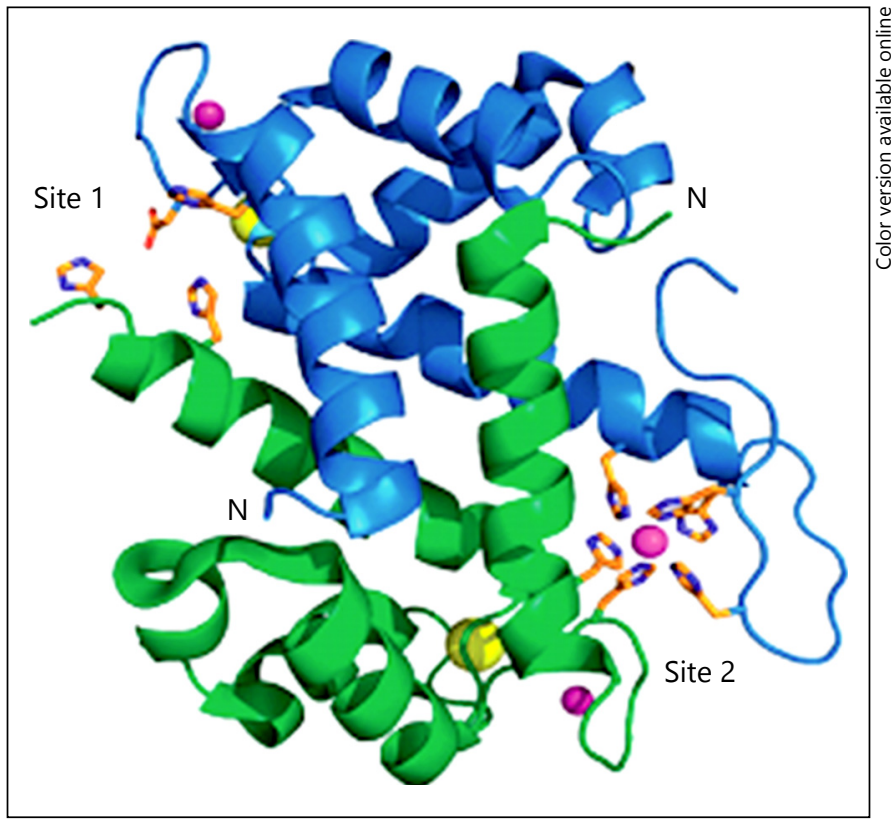

Fig. 1. Calprotectin heterodimer. S100A8 is shown in green and S100A9 in blue. The calcium ions are shown as yellow spheres and sodium ions are shown as purple spheres (colors refer to the online version only) [6].

These markers are however non-specific, as they tend to increase in many systemic inflammatory conditions.

The immune markers p-ANCA (Antineutrophil cytoplasmic antibodies) and ASCA (Anti Saccharomyces cerevisiae antibodies) are known to have an excellent specificity (>90\%) for IBD but a low sensibility (50-60\%), thereby precluding their use as diagnostic tool [3].

The gold standard for diagnosis and assessment of intestinal inflammation remains colonoscopy with biopsy sampling, but it is an invasive and expensive exam that is not popular among the general population.

In 1972, Harris et al. [4] first suggested that faecal leukocytes resulted from a breach in the colonic mucosa and since then faecal leucocyte testing (FLT) has been used to evaluate the presence of mucosal inflammation and disruption. Today, FLT is mainly used to screen for infectious diarrhoea. However, FLT has a poor performance in distinguishing infectious and non-infectious intestinal inflammation, and remains above all highly non-specific. Moreover, it has a great variability in stool samples, probably due to the fact that faecal leukocytes degenerate if they are not processed quickly (within one hour after stools collection). Therefore, inflammatory processes involving the right colon may go undetected and a falsenegative FLT may occur [5].
The ${ }^{111}$ Indium or ${ }^{99}$ Technetium labelled granulocytes excretion tests is considered a very accurate technique for detecting intestinal inflammation in research settings, but it requires a complex granulocytes extraction technique over several days, making it cumbersome and impractical in daily routine.

In recent years, new biomarkers have been strenuously sought, which should ideally display extreme accuracy, high reproducibility and ease of dosing. To this end, faecal calprotectin (FC) seems the ideal candidate. FC is an easy test to perform and appears economical, non-invasive and acceptable by patients.

\section{Faecal Calprotectin}

Calprotectin is a calcium-binding protein (heterodimer S100A8/A9), classified as damage associated molecular pattern protein, having antimicrobial properties (Fig. 1) [6]. It accounts for $60 \%$ of neutrophil cytosolic content and it is also found in monocytes and macrophages [7]. The accumulation of neutrophils in the mucosa, a feature of inflammatory flares, results in the release of FC in the stools, where it can be easily measured. FC excretion has been shown to correlate well with ${ }^{111}$ Indium test [8]. Calprotectin is not subject to proteolytic degradation in faeces and remains stable at room temperature for 3-7 days. The variability inevitably found in calprotectin concentration does not reduce its clinical utility and no dietary substance has been found to interfere with the assays. Regular use of anti-inflammatory agents (NSAIDS) may cause enteropathy in some individuals influencing FC concentration; therefore, it is recommended to stop NSAIDS several weeks before FC stool measurement. Calprotectin is measured by ELISA assays and a number of commercial kits are available on the market (Table 1). Several studies have evaluated the semiquantitative and qualitative point-of-care assays and the results are comparable to that of ELISA testing [9]. The normal range for FC is considered $<50 \mu \mathrm{g} / \mathrm{g}$ of faeces; however, there is a great variability in healthy population depending on patients' age. Calprotectin level is higher in paediatric age, that is, before 5 years (when it starts aligning with adults values), probably because of an increased permeability in intestinal mucosa and differences in intestinal flora. FC for children from 2 to 9 years is considered normal if $<166 \mu \mathrm{g} / \mathrm{g}$, in adults aged between 10 and 59 if $<51 \mu \mathrm{g} / \mathrm{g}$ and it drops at $<112 \mu \mathrm{g} / \mathrm{g}$ after 60 years [10]. In 2004, Poullis et al. [11] assessed the relation between bowel inflammation in the general population aged 50- 
Table 1. Characteristics of the most used calprotectin assays

\begin{tabular}{|c|c|c|c|}
\hline Assay (manufacturer) & Method & Extraction device & Measurement range, $\mu \mathrm{g} / \mathrm{g}$ \\
\hline Calprest (Eurospital, Trieste, Italy) & ELISA & Eurospital extraction device & $15-500$ \\
\hline $\begin{array}{l}\text { Calprotectin EliA (Phadia/Thermo- } \\
\text { Fisher, Uppsala, Sweden) }\end{array}$ & Enzyme fluoroimmuno assay & Roche faecal extraction device & $15-30,000$ \\
\hline $\begin{array}{l}\text { Calprotectin quantum blue } \\
\text { (Buhlmann, Schonenbuch, } \\
\text { Switzerland) }\end{array}$ & $\begin{array}{l}\text { Quantitative immunochrom } \\
\text { atography (automated reading) }\end{array}$ & Roche faecal extraction device & $30-300$ \\
\hline Certest (Biotec, Zaragoza, Spain) & $\begin{array}{l}\text { Semi-quantitative immunochrom } \\
\text { atography (visual reading) }\end{array}$ & Certest extraction device & $50-200$ \\
\hline $\begin{array}{l}\text { Prevent ID caldetect (preventis } \\
\text { GmbH, Bensheim, Germany) }\end{array}$ & $\begin{array}{l}\text { Semi quantitative immunochrom } \\
\text { atography rapid test }\end{array}$ & $\begin{array}{l}\text { PreventID }{ }^{\circledR} \text { CalDetect }^{\circledR} \text { test } \\
\text { device }\end{array}$ & $15-16$ \\
\hline
\end{tabular}

70 and lifestyle risk factors for colorectal carcinoma and found a significant positive relationship between FC and increasing age (Table 2).

\section{Discrimination between Functional and Organic Disease}

IBD and IBS share many symptoms, such as bloating, altered bowel habits and malaise and it is not easy for gastroenterologists to formulate a diagnosis on clinical presentation solely. To this end, Rome III criteria were introduced as a mean of cost-effective management of patients with GI symptoms. Indeed, the main concern for physicians is to avoid misdiagnosing an organic disease leading to a potential delay in treatment. Until recently, the only reliable complementary exam to distinguish between IBS and IBD was endoscopy with biopsy sampling, with all subsequent implications for health care costs as well as the risk of exposing patients to unnecessary invasive procedures.

In 2002, Tibble et al. [12] prospectively studied 602 consecutive patients referred to a gastroenterology outpatient department, all with symptoms suggestive of either IBD or IBS. All patients underwent invasive diagnostic imaging procedures (barium enteroclysis/enema and/
Table 2. Relationship between age and FC mean levels (Poullis et al. [11])

\begin{tabular}{ll}
\hline Age, range & Mean FC, $\mu \mathrm{g} / \mathrm{g}$ \\
\hline $50-54$ years & 27 \\
$55-59$ years & 28 \\
$60-64$ years & 26 \\
$65-70$ years & 45 \\
\hline
\end{tabular}

or endoscopy), whereas FC was measured in each patient. The FC levels in all organic diagnostic groups differed significantly from the non-organic group. With a cut-off value of $10 \mathrm{mg} / \mathrm{L}$ (equivalent of $50 \mu \mathrm{g} / \mathrm{g}$ ), the sensitivity and specificity of calprotectin for organic disease were 89 and $79 \%$ respectively, with a positive predictive value $(\mathrm{PPV})$ at 0.76 and a negative predictive value (NPV) at 0.89 . A recent meta-analysis [13] included a total of 28 studies including both adults and children, analyzing the role of FC in different settings: differentiation of IBD and IBS, organic disease versus IBS, IBD versus non-inflammatory bowel disease and organic versus non-organic bowel disease. In adults, 7 studies were analyzed for differentiating IBD and IBS. All studies were performed in secondary care centres in which rapid ELISA assays were 
used. These studies gave pooled sensitivity of $93 \%$ and specificity of $94 \%$ at an FC cut-off level of $50 \mu \mathrm{g} / \mathrm{g}$. Sensitivities at that specific cut-off ranged from 83 to $100 \%$, whereas specificities ranged from 60 to $100 \%$. This metaanalysis showed that FC had a high sensitivity and PPV ranged between $0.20-1$ and NPV between $0.73-1$. These results concluded that dosing FC has a low risk of giving false negative results (that is of missing a diagnosis of IBD), and a higher risk of false positive, that is, to perform an unnecessary endoscopy to patients with functional disease. In 2008, Schoepfer et al. [14] compared the test performance of FC, lactoferrin, blood leucocytes, CRP and IBD antibodies (ANCA and ASCA). They prospectively included 64 patients with IBD (36 CD, $28 \mathrm{UC}), 30$ with IBS and 42 healthy controls. The overall accuracy of tests for discriminating IBD from IBS was $90 \%$ for lactoferrin, $89 \%$ for FC (PhiCal Test), 73\%, for CRP, 63\% for blood leukocytes, $55 \%$ for CD antibodies (ASCA+/pANCA- or ASCA+/pANCA+) and 49\% UC antibodies (pANCA+/ASCA-). The overall accuracy of IBD-SCAN (lactoferrin) and PhiCal test combined with ASCA/pANCA for discriminating IBD from IBS was 92 and $91 \%$ respectively.

\section{FC and Evaluation of IBD Activity}

The follow-up of patients with IBD might be difficult and until recently gastroenterologists had few tools at their disposal but for the activity scores or endoscopy scores. Activity scores combine subjective symptoms, objective findings in clinical evaluation and laboratory values. These scores represent more the patient's subjective well-being than the degree of mucosal inflammation and they are limited by poor reproducibility. For these reasons, activity scores are mainly used in clinical studies and not in clinical practice. FC has found its role in monitoring the activity of inflammatory diseases. Sipponen et al. [15] in 2008 correlated the FC concentration with the CDAI and the endoscopic index of severity (Crohn's Disease Index of Severity [CDEIS]) in 77 CD patients. FC correlated significantly with CDEIS and using a cut-off level of $200 \mu \mathrm{g} / \mathrm{g}$, sensitivity was $70 \%$, specificity $92 \%$, PPV 94\% and NPV 61\% in predicting endoscopically active disease (CDEIS $\geq 3$ ). Since then other studies confirmed these findings [15-17].

Since mucosal healing has become a treatment goal in IBD, D'Haens et al. [17] examined how reliably FC levels reflect mucosal disease activity. In this study, 126 IBD patients and 32 IBS patients needing colonoscopy were in- cluded. In all patients, the FC level was measured before endoscopy and endoscopic scores were recorded (SESCD and CDEIS in CD patients and the Mayo endoscopic score in UC patients). Correlations between FC levels and endoscopic disease scores were significant in both CD and in UC. In particular, in CD, FC levels $>250 \mu \mathrm{g} / \mathrm{g}$ indicated the presence of large ulcers with a sensitivity of $60.4 \%$ and a specificity of $79.5 \%$ (PPV 78.4\%, NPV 62.0\%); levels $\leq 250 \mu \mathrm{g} / \mathrm{g}$ predicted endoscopic remission (CDEIS $\leq 3$ ) with $94.1 \%$ sensitivity and $62.2 \%$ specificity (PPV $48.5 \%$, NPV 96.6\%). In UC, an FC > $250 \mu \mathrm{g} / \mathrm{g}$ gave a sensitivity of $71.0 \%$ and a specificity of $100.0 \%$ (PPV 100.0\%, NPV 47.1\%) for active mucosal disease activity (Mayo $>0)$.

The correlation between FC and endoscopic activity was also confirmed for UC by Schoepfer et al. [18] in a population of $228 \mathrm{UC}$ patients and 52 healthy controls. Endoscopic disease activity according to the modified Baron Index best correlated with FC compared to CRP, platelets count, hemoglobin, blood leukocytes count, and the Lichtiger Index (clinical score). FC with a cut-off at $57 \mu \mathrm{g} / \mathrm{g}$ had a sensitivity of $91 \%$ and a specificity of $90 \%$ to detect an endoscopically active disease (modified Baron Index $\geq 2$ ). Moreover, FC was the only marker that could discriminate between different grades of endoscopic activity (grade 0 , mean of $16 \mu \mathrm{g} / \mathrm{g}$; grade 1,35 $\mu \mathrm{g} / \mathrm{g}$; grade $2,102 \mu \mathrm{g} / \mathrm{g}$; grade $3,235 \mu \mathrm{g} / \mathrm{g}$; grade 4,611 $\mu \mathrm{g} / \mathrm{g})$.

Some studies demonstrated that FC can also be used as a marker of subclinical inflammation. Thus, it has an interesting role for patients who remain in clinical remission but still present an inflammatory activity in bowel mucosa, therefore being at risk for subsequent relapses. Tibble et al. [18] found that the sensitivity and specificity of calprotectin in predicting relapse in all patients with IBD were 90 and $83 \%$ respectively. These findings were found to be true for both CD and UC. A prospective multicentre study in 2009 included 163 patients (89 CD, 74 UC) who had been in clinical remission for 6 months, and followed up for 12 months. The FC was evaluated at baseline, at time of relapses and at the end of follow-up. Calprotectin concentrations in patients who suffered a relapse were higher than in non-relapsing patients (239 \pm 150 vs. $136 \pm 158 \mu \mathrm{g} / \mathrm{g} ; p<0.001)$. Relapse risk was higher in patients having high $(>150 \mu \mathrm{g} / \mathrm{g})$ calprotectin concentrations (30 vs. $7.8 \%$; $p<0.001$ ). Faecal calprotectin $(>150 \mu \mathrm{g} / \mathrm{g})$ sensitivity and specificity to predict relapse were 69 and $69 \%$, respectively, but 100\% sensitivity was obtained when only colonic CD disease or only relapses during the first 3 months were considered [19]. FC seems 
to be less reliable in predicting flares in patients with ileal CD compared to patients with UC or colonic/ileo-colonic CD [20].

\section{FC to Monitor Treatment Response}

Several studies have demonstrated a role for FC to monitor treatment response in IBD, both for CD and UC. Wagner et al. [21] included in their study 38 patients with IBD (27 with UC and 11 with CD) who were evaluated at baseline and after 4 and 8 weeks of treatment. At inclusion, $97 \%$ of patients had elevated FC levels ( $>94.7$ $\mu \mathrm{g} / \mathrm{g})$. Patients were evaluated with clinical and endoscopic scores with histopathology assessments. In UC, a complete response to treatment was defined as the return of the clinical and endoscopic scores to normal, and a partial response was defined as reduction in both the clinical and endoscopic scores, but their failure to return to normal. In CD, a complete response was defined as a decrease in the Harvey-Bradshaw's clinical activity index score to $\leq 5$ points, and partial response was a decrease in the clinical score but not $<6$ points. At the end of the study, $82 \%$ patients fulfilled criteria of a complete response (UC 21/27 [78\%]; CD 10/11 [91\%]). Overall, a normalised FC level at the end of the study predicted a complete response in $100 \%$ patients, whereas elevated FC levels predicted incomplete response in 30\%. Similar results were found by Sipponen et al. [22] investigating patients with $\mathrm{CD}$ on anti-TNF alpha treatment. Before and 3 months after the beginning of anti-TNF-alpha induction, patients underwent ileocolonoscopy with scoring of the CDEIS and FC measurement. In patients showing an endoscopic response to treatment or endoscopic remission (CDEIS <3), FC concentration declined accordingly with significantly lower levels post treatment. Recently, Lasson et al. [23] performed an interventional study to evaluate whether pharmacological intervention guided by FC concentration prolongs remission in patients with UC. A total of 91 adults with UC in remission were randomized to an intervention group or a control group. Analysis of FC was performed monthly, during 18 months. The patients in the intervention group performed a dose escalation of their ongoing 5-ASA treatment, if the value of FC in the monthly collected stool samples was $>300 \mu \mathrm{g} / \mathrm{g}$.

The primary study end-point was the number of patients to have relapsed by month 18 and authors failed to demonstrate statistically significant differences in overall relapse rates. However, it was demonstrated that patients

Calprotectin in Daily Practice: Where Do

We Stand in 2017? with active intervention experienced fewer disease relapses, as compared with patients in the control group with corresponding FC levels. This study, despite having various limitations (i.e., limited sample size, absence of endoscopic confirmation of flares), shows that FC concentration might be used to identify patients at risk of flares, thus prompting the need to adapt to treatment or refer to colonoscopy.

FC level monitoring was also analysed in the STORI trials, a study aimed to assess the risk of relapse following the withdrawal of Infliximab (IFX) therapy in CD patients in long-term remission. It is a prospective study including $115 \mathrm{CD}$ patients treated for at least 1 year with a double therapy of IFX and an antimetabolite and in remission for at least 6 months. IFX was stopped, and patients were followed up for at least 1 year. After a median follow-up period of 28 months, 52 of the 115 patients experienced a relapse; the 1 -year relapse rate was $43.9 \%$. The authors were able to identify 6 risk factors for relapse including male gender, the absence of surgical resection, leukocyte counts $>6.0 \mathrm{G} / \mathrm{L}$, levels of hemoglobin $<145$ $\mathrm{g} / \mathrm{L}, \mathrm{CRP}>5.0 \mathrm{mg} / \mathrm{L}$, and faecal calprotectin $>300 \mu \mathrm{g} / \mathrm{g}$. Patients with no more than 2 of these risk factors (approximately $29 \%$ of the study population) had a $15 \%$ risk of relapse within 1 year. In this low-risk population group, the cessation of IFX therapy could be contemplated [24]. Roblin et al. [25], in a recent prospective monocentric study developed a predictive model for relapse in CD patients in clinical remission on IFX treatment by including biomarkers used in clinical practice, namely, CRP, FC, trough-levels of IFX (TLI) and IFX antibodies (ATI). One hundred nineteen $\mathrm{CD}$ patients on IFX maintenance treatment $(5 \mathrm{mg} / \mathrm{kg})$ considered in remission $(\mathrm{CDAI}<150)$ for at least 16 weeks were included in the study and then followed up for a minimum of nine months. During follow-up, 37 patients (31.1\%) relapsed, $78 \%$ within the first 6 months. The authors were able to isolate 4 significant factors predicting the loss of response (LOR) to treatment, namely, CRP $>5 \mathrm{mg} / \mathrm{L}(p=0.043)$, ATI $>20 \mathrm{ng} / \mathrm{mL}(p<0.001)$, LTI $>2 \mu \mathrm{g} / \mathrm{mL}(p<0.001)$ and FC $>250 \mu \mathrm{g} / \mathrm{g}(p<0.001)$. Two independent factors (LTI $<2 \mu \mathrm{g} / \mathrm{mL}$ and FC $>250 \mu \mathrm{g} / \mathrm{g}$ ) were linked to a loss of clinical response. In a training cohort of 55 patients isolated randomly, the combination of calprotectin $>250$ $\mu \mathrm{g} / \mathrm{g}$ and $\mathrm{TLI}<2 \mu \mathrm{g} / \mathrm{mL}$ enabled to predict LOR in $95 \%$ of the cases within 6 months [25]. Despite these results being encouraging, this study presents several limitations due to the limited number of patients analysed and the absence of an endoscopic confirmation of mucosal healing. 
The dosage of FC has also been studied and found to predict the need for surgery and the severity of postoperative endoscopic recurrence.

Ho et al. [26] were the first to demonstrate that FC level was dramatically increased in acute severe UC (ASUC) and that this marker could predict the need for further colectomy as well as corticosteroid and IFX nonresponse. Specifically, 90 patients with ASUC requiring intensive in-patient medical therapy were included. All patients were treated with steroids as first-line therapy and IFX as second-line treatment. Out of 90 patients, 31 (34.4\%) required further colectomy, including 11 (52.4\%) of those treated with IFX. Overall FC concentrations were high reaching mean values of $601.5-1,617.5 \mu \mathrm{g} / \mathrm{g}$ ) with levels significantly higher in patients requiring colectomy $(1,200.0$ vs. $887.0 ; p=0.04)$, with a trend towards significance when comparing corticosteroid non-responders and responders $(1,100.0$ vs. $863.5 ; p=0.08)$, and also when comparing IFX non-responders and responders $(1,795.0$ vs. $920.5 ; p=0.06)$. In the POCER study, Boschetti et al. [27] analysed the relationships between the levels of FC and high-sensitivity CRP (hsCRP) and the presence and severity of postoperative endoscopic recurrence in 86 asymptomatic CD patients. hsCRP and FC were measured and a routine ileocolonoscopy was performed within 18 months from resection to detect endoscopic recurrence according to the Rutgeerts score. FC concentrations differed significantly in patients with endoscopic recurrence when compared with those in endoscopic remission. The best cut-off point for FC to distinguish between endoscopic remission and recurrence was $100 \mu \mathrm{g} / \mathrm{g}$ with $95 \%$ sensitivity, $54 \%$ specificity, PPV $69 \%$ and NPV 93\%. According to this study, the measurement of FC concentrations could be a useful tool for monitoring asymptomatic CD patients after ileocolonic resection. Taking into account the high NPV, a threshold of FC below $100 \mu \mathrm{g} / \mathrm{g}$ could avoid systematic ileocolonoscopies in $30 \%$ of patients from the studied population.

\section{Role of FC in CC}

CC is a subtype of microscopic colitis, usually presenting with chronic watery diarrhoea in elderly women. Colonic mucosa appears often normal, but histopathology shows abnormal thickening of the subepithelial collagen layer $(>10 \mu \mathrm{m})$ with lymphocytic infiltration of the epithelium and lamina propria. In addition, eosinophils, neutrophils as well as mast cells and macrophages can be found in tissue samples. In CC concentrations of faecal markers as calprotectin are expected to be low due to the dominant mononuclear inflammation. However, in the literature results are contradicting. Wildt et al. [28] analysed faecal samples from 21 patients with active CC, 12 patients in remission, and 13 controls. Calprotectin concentrations were significantly increased in patients with active CC (median $80 \mu \mathrm{g} / \mathrm{g}$ ) compared with patients with quiescent CC (median $26 \mu \mathrm{g} / \mathrm{g}$ ) and controls (median $6.25 \mu \mathrm{g} / \mathrm{g}$ ). However, more than one third (38\%) of patients with active CC had normal levels of calprotectin. The high levels of FC in active CC patients can be explained by the fact that calprotectin is present not only in neutrophils but also in monocyes and macrophages. Nevertheless, the use of FC as an inflammatory marker for CC is not recommended for lack of universal evidence.

\section{Role of FC in Infectious Colitis}

Infectious colitis should be suspected in patients with diarrhoea and where there is evidence of colonic inflammation, including fever, dysentery, mucosal inflammation at colonoscopy or presence of faecal inflammatory markers (leucocytes, calprotectin or lactoferrin). The faecal leucocyte test looks for the presence of white blood cells (WBC) in stools, providing semi-quantitative information (few, moderate, many). Many WBC are often a sign of diffuse colonic inflammation. However, in case of focal colitis, WBC will be either absent or few in numbers because of their dilution in luminal content. The finding of many WBC does not differentiate between infectious colitis and IBD [29]. The first study to use FC as diagnostic tool for acute bacterial diarrhoea was a prospective case-control multicentre study performed in Germany by Shastri et al. [30] during 3 years (2004-2007). A total of 2,383 consecutive patients presenting with acute diarrhoea were included and stool cultures were performed in each patient. Then, an equal number of patients with positive cultures was matched with an equal number of controls with negative cultures, with both groups undergoing faecal occult blood test, calprotectin and lactoferrin assays. Calprotectin showed high correlation with bacteriologically positive infectious diarrhoea compared with lactoferrin and faecal occult blood test, with sensitivity and specificity being 83 and $87 \%$ respectively [29]. A study conducted in children with acute gastroenteritis showed that FC levels were higher in cases of bacterial etiology compared with viral etiology. FC was the best- 
rated marker compared to bowel movements, fever, ESR, WBC count and CRP, with a diagnostic accuracy of $92 \%$ [31]. Despite these encouraging results, few studies are available at present on the use of FC in infectious colitis and more data are needed to confirm the role of calprotectin measurement in this setting. In general, finding inflammatory markers in stools of patients with acute diarrhoea is considered an indication to perform stool cultures to identify an infectious cause or to perform a colonoscopy when IBD is suspected.

\section{Calprotectin: Strong Points and Limits}

FC occupies an important place in routine clinical practice. Is it an economic, non-invasive tool, well tolerated by patients and can be considered a reliable marker of intestinal inflammation with good clinical sensitivity in differentiating organic and functional disease. FC has been proved to be useful in the follow-up of IBD patients, even in cases of clinical remission, as CF levels raise during flares. Moreover, the persistence of high levels of calprotectin despite treatment represents a risk factor for resistance to treatment and for potentially coming flares.

In the last few years, there has been increasing interest in achieving mucosal healing in patients with IBD. It was demonstrated that the severity of mucosal inflammation correlates with the number of relapses, number of hospitalisations, need for surgery and cancer occurrence [32]. FC has been shown to have an excellent correlation with endoscopical assessment, better than all serological markers or clinical indices used so far.

Although calprotectin is a very sensitive marker for detection of inflammation in the gastrointestinal tract, it is not a specific marker and increased levels are also found in colorectal cancer, infections, and polyps. The same is true for NSAIDs use. In a recent prospective study, the intra-individual variability of FC was evaluated. For 2 days, 18 patients with active UC collected 2 stool samples at each bowel movement, for a total of 287 stool samples. The median individual coefficient of variation in samples collected during the same day was $52 \%$ and the levels of FC increased with longer time between the bowel movements. However, it is important to stress that the variation was greatest in patients with high levels of FC and the variation increased with higher concentrations of FC, thus limiting the clinical relevance [33].

Rarely, some people with IBS were found to be positive on calprotectin testing, suggesting that IBS may have an inflammatory component. It has been suggested that this may be due to disturbances in the intestinal bacterial flora, followed by a mucosal response. The high calprotectin levels seen in some people with IBS are perhaps not surprising, as raised levels have been reported in a random sample of the general population by Poullis et al. [11] In this study involving people aged 50-70, the authors found that one-quarter of the population had calprotectin levels above the upper limit of $65 \mu \mathrm{g} / \mathrm{g}$. Some recruits may have had colonic adenomas, which are common in this age range, but no data was provided, as no colonoscopy was performed.

FC measurement does not differentiate between CD, UC, or CC; neither does it give any information on the localisation of the disease.

Another concern with FC measurement is the lack of a clear cut-off level and this applies not only to differentiate organic and functional disease but also to evaluate IBD activity and to monitor treatment response. The decision of the cutoff depends on different parameters such as the type of assay, recommendations of manufacturer, published data and clinical setting. Most observers believe that levels $<50 \mu \mathrm{g} / \mathrm{g}$ might be considered negative, whereas levels between 50 and $100 \mu \mathrm{g} / \mathrm{g}$ could be considered weakly positive, and $>100 \mu \mathrm{g} / \mathrm{g}$ as highly positive. For patients with FC in the intermediate range $50-150 \mu \mathrm{g} / \mathrm{g}$, the clinician will evaluate whether an endoscopy is indicated or whether he shall repeat the FC test, bearing in mind the risk of diagnostic delay. In newly diagnosed patients with CD or UC, it is common for FC to exceed 1,000 $\mu \mathrm{g} / \mathrm{g}$. A good response to treatment may lower this value to $500 \mu \mathrm{g} / \mathrm{g}$.

\section{FC in Daily Clinical Routine}

National Institute for Clinical Excellence (NICE) guidelines published in 2008 [34] state that patients presenting with symptoms of abdominal pain/discomfort, bloating or change in bowel habit should be investigated for any "red flag" indicators including unexplained weight loss, rectal bleeding, family history of rectal or ovarian cancer, change in bowel habit, anaemia, abdominal or rectal mass and elevated inflammatory markers. The presence of any "red flag" indicator should promptly lead to refer the patient to secondary care centre for further investigation including colonoscopy. In cases where "red flag" indicators are not present, a simple noninvasive test such as FC has the potential to reduce waiting lists for colonoscopy and reduce costs. The most re- 


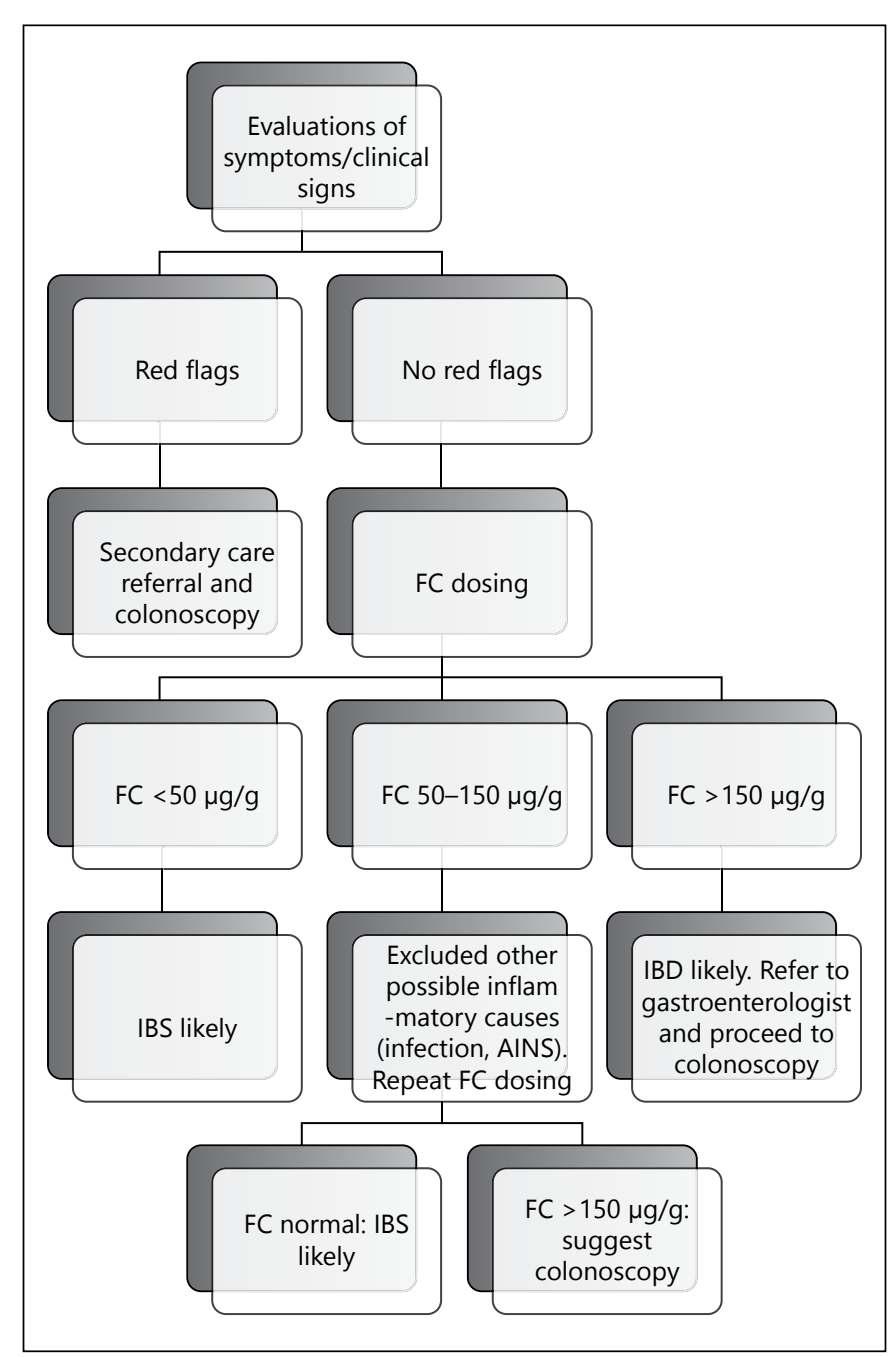

Fig. 2. Algorithm for the use of FC measurement in differential diagnosis between IBS and IBD.

cent NICE guidelines published in 2013 recommend FC testing as an option to support clinicians in their differential diagnosis between IBD and IBS in adults with recent onset of lower gastrointestinal symptoms. These guidelines may be used for patients for whom specialist assessment is being considered in case cancer is not suspected, and when appropriate quality assurance processes and locally agreed care pathways are available for testing [35]. A diagnostic algorithm is proposed in Figure 2 [36]. FC has its role in monitoring IBD activity and assessing treatment response in active IBD. FC can increase before symptoms of flare appear and it may prompt a modification of treatment or a referral to endoscopy. To monitor disease activity, FC serial measurements are preferred and the development of point-of-care testing

has opened the doors to a better patient follow-up. In 2015, therapeutic targets in IBD recommendations aimed at a treat-to-target strategy were published. The objective was to achieve potential treatment targets for IBD based on an expert consensus that could be used in daily clinical practice. Available biomarkers, including FC, are not considered targets, but adjunctive measures of monitoring inflammation. According to authors, there is not sufficient evidence to recommend treatment optimization by taking into account biomarkers alone. Failure of FC normalization should then prompt an endoscopic evaluation irrespective of symptoms [37]. Important studies, such as the CALM study (NCT01235689completed in November 2016), have been developed to demonstrate that tight control of the disease activity in $\mathrm{CD}$ using stringent criteria based on CDAI, CRP and FC improves the rate of mucosal healing as compared to less stringent criteria. The results are still pending, but the overall international literature is supporting this treatto-target approach in the hope that the use of prognostic and predictive biomarkers may be effective in improving patients monitoring, management and eventually disease control.

\section{Disclosure Statement}

The authors report no conflicts of interest.

\section{Funding}

No specific funding has been received for this work.

References

Digestion 2017;95:293-301 DOI: $10.1159 / 000476062$
1 Keohane J, O’Mahony C, O’Mahony L, O’Mahony S, Quigley EM, Shanahan F: Irritable bowel syndrome-type symptoms in patients with inflammatory bowel disease: a real association or reflection of occult inflammation? Am J Gastroenterol 2010;105:1788, 1789-1794; quiz 1795.

2 Vavricka SR, Spigaglia SM, Rogler G, Pittet V, Michetti P, Felley C, Mottet C, Braegger CP, Rogler D, Straumann A, Bauerfeind P, Fried M, Schoepfer AM; Swiss IBD Cohort Study Group: Systematic evaluation of risk factors for diagnostic delay in inflammatory bowel disease. Inflamm Bowel Dis 2012;18:496-505.

3 Peeters M, Joossens S, Vermeire S, Vlietinck $\mathrm{R}$, Bossuyt X, Rutgeerts P: Diagnostic value of anti-Saccharomyces cerevisiae and antineutrophil cytoplasmic autoantibodies in inflammatory bowel disease. Am J Gastroenterol 2001;96:730-734. 
4 Harris JC, Dupont HL, Hornick RB: Fecal leukocytes in diarrheal illness. Ann Intern Med 1972;76:697-703.

5 Granville LA, Cernoch P, Land GA, Davis JR: Performance assessment of the fecal leukocyte test for inpatients. J Clin Microbiol 2004; 42:1254-1256.

6 Nakashige T, Zhang B, Krebs C, Nolan E: Human calprotectin is an iron-sequestering host-defense protein. Nat Chem Biol 2015;11: 765-771.

7 Steinbakk M, Naess-Andresen CF, Lingaas E, Dale I, Brandtzaeg P, Fagerhol MK: Antimicrobial actions of calcium binding leucocyte L1 protein, calprotectin. Lancet 1990;336: 763-765.

8 Tibble JA, Sigthorsson G, Bridger S, Fagerhol MK, Bjarnason I: Surrogate markers of intestinal inflammation are predictive of relapse in patients with inflammatory bowel disease. Gastroenterology 2000;119:15-22.

9 Sherwood R: Fecal markers of gastrointestinal inflammation. J Clin Pathol 2012;0:1-5.

10 Joshi S, Lewis S, Creanor S, Ayling R: Agerelated faecal calprotectin, lactoferrin and tumour M2-PK concentrations in healthy volunteers. Ann Clin Biochem 2010;47(pt 3): 259-263.

11 Poullis A, Foster R, Shetty A, Fagerhol MK, Mendall MA: Bowel inflammation as measured by fecal calprotectin: a link between lifestyle factors and colorectal cancer risk. Cancer Epidemiol Biomarkers Prev 2004;13:279284.

12 Tibble JA, Sigthorsson G, Foster R, Forgacs I, Bjarnason I: Use of surrogate markers of inflammation and Rome criteria to distinguish organic from nonorganic intestinal disease. Gastroenterology 2002;123:450-460.

13 Waugh N, Cummins E, Royle P, Kandala NB Shyangdan D, Arasaradnam R, Clar C, Johnston R: Faecal calprotectin testing for differentiating amongst inflammatory and non-inflammatory bowel diseases: systematic review and economic evaluation. Health Technol Assess 2013;17:xv-xix, 1-211.

14 Schoepfer AM, Trummler M, Seeholzer P, Seibold-Schmid B, Seibold F: Discriminating IBD from IBS: comparison of the test performance of fecal markers, blood leukocytes, CRP, and IBD antibodies. Inflamm Bowel Dis 2008; 14:32-39.

15 Sipponen T, Savilahti E, Kolho KL, Nuutinen $\mathrm{H}$, Turunen U, Färkkilä M: Crohn's disease activity assessed by fecal calprotectin and lactoferrin: correlation with Crohn's disease activity index and endoscopic findings. Inflamm Bowel Dis 2008;14:40-46.

16 Sipponen T, Kärkkäinen P, Savilahti E, Kolho KL, Nuutinen H, Turunen U, Färkkilä M:
Correlation of faecal calprotectin and lactoferrin with an endoscopic score for Crohn's disease and histological findings. Aliment Pharmacol Ther 2008;28:1221-1229.

17 D'Haens G, Ferrante M, Vermeire S, Baert F, Noman M, Moortgat L, Geens P, Iwens D, Aerden I, Van Assche G, Van Olmen G, Rutgeerts $\mathrm{P}$ : Fecal calprotectin is a surrogate marker for endoscopic lesions in inflammatory bowel disease. Inflamm Bowel Dis 2012; 18:2218-2224.

18 Schoepfer AM, Beglinger C, Straumann A, Safroneeva E, Romero Y, Armstrong D, Schmidt C, Trummler M, Pittet V, Vavricka SR: Fecal calprotectin more accurately reflects endoscopic activity of ulcerative colitis than the Lichtiger Index, C-reactive protein, platelets, hemoglobin, and blood leukocytes. Inflamm Bowel Dis 2013;19:332-341.

19 Gisbert JP, Bermejo F, Pérez-Calle JL, Taxonera C, Vera I, McNicholl AG, Algaba A, López P, López-Palacios N, Calvo $M$, González-Lama Y, Carneros JA, Velasco M, Maté J: Fecal calprotectin and lactoferrin for the prediction of inflammatory bowel disease relapse. Inflamm Bowel Dis 2009;15:11901198.

20 D'Incà R, Dal Pont E, Di Leo V, Benazzato L, Martinato M, Lamboglia F, Oliva L, Sturniolo GC: Can calprotectin predict relapse risk in inflammatory bowel disease? Am J Gastroenterol 2008;103:2007-2014.

21 Wagner M, Peterson CG, Ridefelt P, Sangfelt $\mathrm{P}$, Carlson M: Fecal markers of inflammation used as surrogate markers for treatment outcome in relapsing inflammatory bowel disease. World J Gastroenterol 2008;14:55845589; discussion 5588

22 Sipponen T, Savilahti E, Kärkkäinen P, Kolho KL, Nuutinen H, Turunen U, Färkkilä M: Fecal calprotectin, lactoferrin, and endoscopic disease activity in monitoring anti-TNF-alpha therapy for Crohn's disease. Inflamm Bowel Dis 2008;14:1392-1398.

23 Lasson A, Ohman L, Stotzer P, Isaksson S, Uberbacher O, Ung K, Strid H: Pharmacological intervention based on fecal calprotectin levels in patients with ulcerative colitis at high risk of a relapse: a prospective, randomized, controlled study. United European Gastroenterol J 2015;3:72-79.

24 Louis E, Mary JY, Vernier-Massouille G, et al: Groupe D'études Thérapeutiques Des Affections Inflammatoires Digestives: Maintenance of remission among patients with Crohn's disease on antimetabolite therapy after Infliximab therapy is stopped. Gastroenterology 2012;142:63-70.

25 Roblin X, Duru G, Williet N, Del Tedesco E, Cuilleron M, Jarlot C, Phelip JM, Boschetti G,
Flourié B, Nancey S, Peyrin-Biroulet L, Paul S: Inflamm Bowel Dis 2017;23:126-132.

26 Ho GT, Lee HM, Brydon G, Ting T, Hare N, Drummond H, Shand AG, Bartolo DC, Wilson RG, Dunlop MG, Arnott ID, Satsangi J: Fecal calprotectin predicts the clinical course of acute severe ulcerative colitis. Am J Gastroenterol 2009;104:673-678.

27 Boschetti G, Laidet M, Moussata D, Stefanescu C, Roblin X, Phelip G, Cotte E, Passot G Francois Y, Dray J, Del Tedesco E, Bouhnik Y, Flourie B, Nancey S: Levels of fecal calprotectin are associated with the severity of postoperative endoscopic recurrence in asymptomatic patients with Crohn's disease. Am J Gastroenterol 2015;110:865-872.

28 Wildt S, Nordgaard-Lassen I, Bendtsen F, Rumessen JJ: Metabolic and inflammatory faecal markers in collagenous colitis. Eur J Gastroenterol Hepatol 2007;19:567-574.

29 DuPont HL: Approach to the patient with infectious colitis. Curr Opin Gastroenterol 2012;28:39-46.

30 Shastri YM, Bergis D, Povse N, Schäfer V, Shastri S, Weindel M, Ackermann H, Stein J: Prospective multicenter study evaluating fecal calprotectin in adult acute bacterial diarrhea. Am J Med 2008;121:1099-1106.

31 Sýkora J, Siala K, Huml M, Varvařovská J, Schwarz J, Pomahačová R: Evaluation of faecal calprotectin as a valuable non-invasive marker in distinguishing gut pathogens in young children with acute gastroenteritis. Acta Paediatr 2010;99:1389-1395.

32 Pineton de Chambrun G, Peyrin-Biroulet L, Lémann M, Colombel JF: Clinical implications of mucosal healing for the management of IBD. Nat Rev Gastroenterol Hepatol 2010;7:15-29.

33 Lasson A, Stotzer PO, Öhman L, Isaksson S, Sapnara M, Strid H: The intra-individual variability of faecal calprotectin: a prospective study in patients with active ulcerative colitis. J Crohns Colitis 2015;9:26-32.

34 National Institute for Clinical Excellence: Irritable Bowel Syndrome in Adults: Diagnosis and Management of Irritable Bowel Syndrome in Primary Care [Internet]. London, NICE, 2008.

35 National Institute for Clinical Excellence: Faecal Calprotectin Diagnostic Tests for Inflammatory Diseases of the Bowel. NICE Diagnostics Guidance, 2013.

36 Sherwood RA: Faecal markers of gastrointestinal inflammation. J Clin Pathol 2012;65: 981-985.

37 Peyrin-Biroulet L, Sandborn W, Sands BE et al: Selecting therapeutic targets in inflammatory bowel disease (STRIDE): determining therapeutic goals for treat-to-target. Am J Gastroenterol 2015;110:1324-1338. 